\title{
Effects of dietary CP and amino acid restriction on the growth dynamics of organs and body components in entire male, castrated and female pigs
}

\author{
I. Ruiz-Ascacibar ${ }^{1,2}$, P. Stoll ${ }^{1}$ and G. Bee ${ }^{1 \dagger}$ \\ ${ }^{1}$ Agroscope, Tioleyre 4, 1725 Posieux, Switzerland; ${ }^{2}$ ETH Zurich, Institute of Agricultural Sciences, Universitätsstrasse 2, 8092 Zurich, Switzerland
}

(Received 29 January 2018; Accepted 30 November 2018; First published online 26 February 2019)

The optimized use of dietary nutrients and the accurate knowledge of the growth dynamics of body components is important for efficient pig production. This study aimed at evaluating the growth of carcass components and organs of Swiss Large White pigs from birth to $140 \mathrm{~kg} B W$ depending on the $C P$ and amino acid (AA) supply. A total of 66 entire males (EM), 58 castrates (CA) and 66 female (FE) pigs were used. From $20 \mathrm{~kg}$ BW onwards, they had either ad libitum access to a control (C) or a diet (LP) with $20 \%$ lower $C P$, lysine, methionine + cystine, threonine and tryptophan content compared to $C$. The weight of organs, primal cuts and external fat were recorded in eight EM and eight FE; at $10 \mathrm{~kg} \mathrm{BW}$, on two EM, CA and FE each, and at $20 \mathrm{~kg} B W$, on eight pigs from each sex. From 40 to $140 \mathrm{~kg} B W$ at $20 \mathrm{~kg}$ intervals, measurements were recorded on four pigs per sex and dietary treatment. The weight of each component was related to empty body $(E B)$ using allometric regressions. Kidneys were heavier $(\mathrm{P}<0.05)$ in $C$ - than $L P$-pigs and in EM than CA and FE. Above $21 \mathrm{~kg} E B$ weight, growth rate of $L P$-FE overpassed $(P<0.05)$ the one of C-pigs. Consequently, LP-FE had heavier $(P<0.05)$ lean cuts than C-pigs in the finisher period. However, $L P-C A$ and $L P$-EM displayed lower $(\mathrm{P}<0.05)$ weights and growth rates of the lean cuts than C-CA and C-EM. Shoulder and loin weights and growth rates were reduced $(\mathrm{P}<0.05)$ in $L P$-pigs when compared to $C$-pigs. Growth rates of the ham were greater $(P<0.05)$ in $L P$-FE than $C-F E$, whereas in $L P$-EM and $L P$-CA they were lower $(P<0.05)$ than their $C$-counterparts. Total amounts of subcutaneous fat, backfat, ham fat and shoulder fat were lower $(P<0.05)$ in $C$ - than $L P$-pigs. The total amount of subcutaneous fat, backfat and shoulder fat of $C-C A$ was, regardless of diet, greater $(P<0.05)$ than of $C-F E$. In the $L P$ group, this difference was even more pronounced. The amount of deposited ham fat was greater $(P<0.05)$ in $L P$-CA than $L P-F E$, but not in $C-C A v$. $C$-FE. Differences in kidney weights suggested a greater nitrogen clearance required in C-pigs. Overall, dietary restriction and sex did not affect all body parts to the same extent. This study further suggests the possibility to reduce the CP and AA supply in FE without compromising the yield of primal lean cuts or increasing the amount of subcutaneous fat.

Keywords: allometric growth, carcass composition, organs, primal cuts, protein supply

\section{Implications}

Detailed knowledge in terms of carcass cut composition is of considerable importance in the meat industry because each carcass cut has a different saleable market value. In this study, weight and growth rate changes of organs and carcass cuts over a large BW span were monitored in pigs fed two dietary protein and amino acid (AA) levels. The results show that allometric growth of organs, lean meat, and fat cuts differ among cuts and organs depending on the diet and sex. In the area of livestock precision farming, these results can contribute to the optimization of production strategies.

† E-mail: giuseppe.bee@agroscope.admin.ch

\section{Introduction}

In recent years, consumers' demand for leaner pork cuts has constantly increased (Marcoux et al., 2007). To fulfill this demand, EU pig carcass classification is based on the objective measurements of carcass traits using calibrated devices to predict lean meat percentage (Font-i-Furnols et al., 2016). However, the carcass lean meat percentage gives limited information on the proportion of the primal cuts (Knecht et al., 2016), which differs markedly in terms of saleable value. To improve the production efficiency for an optimal slaughter weight, the accurate knowledge of carcass cuts development is primordial for many stakeholders in pig production, such as breeding companies, pig producers, abattoirs and retailers (Gispert et al., 2007; Knecht and 
Duziński, 2016). Landgraf et al. (2007) found significant differences in growth rates between different carcass cuts, suggesting the need for an estimation of their growth patterns.

Due to increased awareness of environmental issues linked to a greater demand for a sustainable livestock production, current meat production systems are subjected to great challenges (Verbeke et al., 2010). One of the priorities in pig production is the sustainable use of feed sources, especially those rich in CP. In a recent study, Ruiz-Ascacibar et al. (2019) found marked differences in $C P$, fat and $A A$ deposition rates in the empty body (EB) (defined as the sum of carcass, blood, bile, organs, intestines, hairs and hooves) of entire males (EM), castrates (CA) and females (FE) fed two levels of dietary $C P$ and essential $A A$, which suggest differences in the growth rate of body tissues between treatments and sex. Since body chemical composition is linked to different body tissues in the EB (Wiseman et al., 2007), differences in the carcass composition are also expected between sexes and dietary treatments. Numerous studies reported changes in the carcass composition in relation to the diet at a given BW or age (Kerr and Easter, 1995; Gispert et al., 2007; Ruusunen et al., 2007; Mas et al., 2010). However, there is a lack of information regarding changes in growth patterns of primal cuts in relation to the diet. By fitting allometric equations and calculating their first derivatives, model parameters with straightforward biological interpretation for describing the body composition and growth rate over a defined growth period can be calculated (Landgraf et al., 2007).

The first objective of the present study was to monitor the changes in weights of carcass cuts and organs from birth to $140 \mathrm{~kg}$ BW in EM, CA and FE. The second objective was to assess differences in the growth dynamics of carcass cuts and organs among sexes and dietary CP and essential AA supply.

\section{Material and methods}

\section{Animals, experimental diets and design}

Detailed experimental design, management protocol and genetic background of the pigs was previously described by Ruiz-Ascacibar et al. (2017). Briefly, a total of 190 Swiss Large White pigs (66 EM, $58 \mathrm{CA}$ and $66 \mathrm{FE}$ ) originating from the Agroscope Posieux sow herd were slaughtered in two series (series one: July 2012 to April 2013; series two: August 2013 to May 2014). At birth, eight $F E$ and eight EM weighing $1.4 \pm 0.44 \mathrm{~kg}$ (mean $\pm \mathrm{SD}$ ) were slaughtered. Subsequently, at $8.9 \pm 0.20 \mathrm{~kg}$ and $20.8 \pm 1.66 \mathrm{~kg}$, two $\mathrm{FE}$, two CA, two EM and eight $F E$, eight $C A$, eight $E M$ were sacrificed, respectively. The remaining 144 pigs were equally allotted per sex to one of the six subsequent slaughter weight categories (40, $60,80,100,120$ or $140 \mathrm{~kg} \mathrm{BW}$ ) and assigned to either a control (C) or a low-CP (LP) diet (Supplementary Table S1). The grower (20 to $60 \mathrm{~kg} \mathrm{BW}$ ), finisher I (60 to $100 \mathrm{~kg} \mathrm{BW}$ ) and finisher II diets (100 to $140 \mathrm{~kg} \mathrm{BW}$ ) of C-group were formulated according to the Swiss standard feeding recommendations for pigs weighing 40,80 and $120 \mathrm{~kg} \mathrm{BW}$ and analyzed CP of 161, 140, $123 \mathrm{~g} / \mathrm{kg}$ (as-fed), respectively (Agroscope, 2017). Compared to the respective $C$ diets, the LP diets were formulated to contain $80 \%$ digestible CP, lysine, methionine + cystine, threonine and tryptophan. To account for their greater protein deposition potential, the grower, finisher I, and finisher II C and LP diets for the EM had $5 \%$ more digestible $\mathrm{CP}$, lysine, methionine + cystine, threonine and tryptophan than those for CA and FE (Agroscope, 2017). All diets were designed to be isocaloric (13.2 MJ digestible Energy $/ \mathrm{kg}$ ). When the individual pig reached the initial BW defined for the grower, finisher I and finisher II period (BW greater than 19,59 and $98 \mathrm{~kg}$, respectively), it was allocated to the following diet: starter to grower, grower to finisher I, finisher I to finisher II. The analyzed composition of the experimental diets has been published by RuizAscacibar et al. (2017) and can be found in the Supplementary Table S1.

\section{Slaughter procedure}

The serial slaughter procedure was used to study the growth dynamics of the different organs and carcass cuts. A description of the slaughter methods is given by RuizAscacibar et al. (2017). Briefly, when the experimental pigs reached their target $\mathrm{BW}$ for slaughter, they were fasted for approximately $16 \mathrm{~h}$, stunned with $\mathrm{CO}_{2}$, and exsanguinated. The semitendinosus muscle from the left carcass side was removed and weighed within $30 \mathrm{~min}$ after exsanguination.

\section{Quantitative organ and carcass measurements}

Carcasses were eviscerated, the head was removed by cutting at the occipital-atlas joint, and then carcasses were split into two halves. The stomach, intestine and hindgut (intestinal tract) were emptied and rinsed with clean water to remove remaining digesta. Before weighing, each of the aforementioned gut components was squeezed by hand to remove excess water. Kidneys, liver and omental fat were also weighted in each animal.

The eviscerated carcasses were chilled overnight at $2^{\circ} \mathrm{C}$ and dissected 1-day postmortem as previously described (Bee et al., 2002). Briefly, the cold left carcass side of each animal was fabricated into ham, loin, shoulder and belly primal cuts. External fat of the loin, ham and shoulder was completely removed. Carcass length $(\mathrm{cm})$ was measured from the base of the tail to the base of the ear.

\section{Calculations and statistical analysis}

The empty gastrointestinal tract (GIT) was considered the sum of the empty stomach, gut and hindgut. The primal cuts were considered the sum of the defatted loin, ham and shoulder. Accordingly, carcass fat was considered the sum of back, ham and shoulder fat. The weight of defatted total lean cuts was the sum of the aforementioned defatted primal cuts expressed as a weight percentage of the left carcass side excluding head, tail, diaphragm and omental fat. Significant differences in the empty BW (EBW) between diets and sex 
within each slaughter weight category were observed. Therefore, a pre-treatment of the data was necessary to normalize the original individual EB organ weight and carcass to standardized EBW categories (Supplementary Material S1). Ranks were then assigned to the transformed data within each BW category from 40 to $140 \mathrm{~kg}$. The ranked data were analyzed using the ANOVA procedure of SYSTAT 13 (SYSTAT Software Inc.) considering the experimental groups (C-EM, C-FE, C-CA, LP-EM, LP-FE, LP-CA), series, and the two-way interactions as fixed effects. To assess whether any of the diet $\times$ sex combinations could be plotted in the same regression equation, the following predefined orthogonal contrasts were tested:

- FE v. CA, fed either C or LP diet

o C-FE v. C-CA

o LP-FE v. LP-CA

- EM pigs $v$. FE/CA, fed either $C$ or LP diet

o C-EM v. C-FE/CA

o LP-EM v. LP-FE/CA

- C diet v. LP diet, regardless of sex

\section{o C-FE/CA per EM v. LP-FE/CA per EM}

Based on the outcome of the orthogonal contrast, the untransformed data of the organ and carcass component weights were grouped and fitted, using the non-linear procedure of SYSTAT 13, to the following allometric regression: $Y=a \times \mathrm{EBW}^{b}$, where $Y$ is the predicted organ or carcass component weight or carcass length, $b$ the scaling exponent or allometric coefficient and $a$ the constant. The weight gain or growth in carcass length, expressed in $\mathrm{g} / \mathrm{kg}$ EBW gain or $\mathrm{cm} / \mathrm{kg}$ EBW gain, were calculated as the first derivative of the aforementioned allometric function for each $\operatorname{EBW}\left(Y^{\prime}=\right.$ $\left.a \times b \times \mathrm{EBW}^{(b-1)}\right)$. The coefficient of determination $\left(R^{2}\right)$ was used to check the goodness of fit. In addition, the residual standard error was used to evaluate the variability in the dependent variable unexplained by the model. The results for the carcass components are presented for the left carcass side.

\section{Results}

\section{Growth performance}

Detailed results on growth performance were presented previously by Ruiz-Ascacibar et al. (2017). Briefly, despite similar feed intake, daily gain and feed efficiency were impaired $(P<0.01)$ from 20 to $100 \mathrm{~kg}$ BW in the LP- compared with the C-pigs. As a consequence, LP-pigs needed more $(P<0.05)$ time than $C$-pigs to achieve the same BW. In the same growth period, CA had the greatest feed intake but, together with $\mathrm{FE}$, displayed the lowest $(P<0.01)$ feed efficiency. Thus, among the three sexes, EM were the most $(P<0.05)$ energy efficient in the grower and finisher I periods.
Average weights of organs, gastrointestinal tract, semitendinosus muscle and carcass cuts

Average weights of kidneys, liver, GIT, lean cuts, belly, omental fat and subcutaneous fat and average length of the carcasses across the BW categories from 40 to $140 \mathrm{~kg}$ are presented for the treatments in Table 1. Average length and weight of each of the aforementioned traits for the pigs at birth, 10 and $20 \mathrm{~kg} \mathrm{BW}$ are reported in Supplementary Tables S2 to S4. Overall, kidneys were heavier $(P<0.05)$ in the $C$ - than LP-group and were heavier $(P<0.05)$ in $\mathrm{EM}$ than $\mathrm{FE}$ and CA pigs. Furthermore, in the LP- but not the $C$-treatment, kidneys were heavier $(P<0.05)$ in $\mathrm{CA}$ than FE. Livers of EM were heavier $(P<0.05)$ than livers of CA and FE when fed the LP-diets. The weight of the GIT was greater $(P<0.05)$ in EM than $\mathrm{FE}$ and $\mathrm{CA}$, regardless of dietary treatment. On average, carcasses were shorter $(P<0.05)$ in $C$ - than LP- and in CA- than FE-pigs. In the LP-, but not the $\mathrm{C}$-group, carcasses of $\mathrm{CA}$ and $\mathrm{FE}$ were shorter $(P<0.05)$ than those of EM. Total primal cuts were $2.4 \%$ heavier in C- than LP-pigs (20.01 v. $19.54 \mathrm{~kg} ; P<0.05)$. When comparing $\mathrm{CA}$ and $\mathrm{FE}$, primal cuts were lighter $(P<0.05)$ in C-CA than C-FE but not in the LP-group. The loins, hams and shoulders were $1.6 \%, 2.8 \%$ and $3.2 \%$ heavier, respectively, in C- than LP-pigs. Regardless of the diet, hams were lighter $(P<0.05)$ in $\mathrm{CA}$ - than FE-pigs. Belly and omental fat weight were lower $(P<0.05)$ in $\mathrm{EM}$ - than $\mathrm{FE}-$ and $\mathrm{CA}$-pigs. Overall belly and omental fat were lighter $(P<0.05)$ in the $C$ - than LP-group and heavier $(P<0.05)$ in CA than FE. The amount of back, ham, shoulder and total subcutaneous fat differed among dietary treatments and sex, resulting in six distinct groups, except for ham fat. Overall, the total amount of subcutaneous, back, ham and shoulder fat was lower $(P<0.05)$ in $C$ - than LP-pigs. Interestingly, C-CA had a $3.4 \%$ greater $(P<0.05)$ amount of subcutaneous fat than C-FE, whereas in the LP-group, this difference was more marked and amounted to $16.5 \%(P<0.05)$. Within dietary treatments, the same difference in the pattern of deposited back and shoulder fat was observed between CAand FE-pigs. The amount of deposited ham fat was greater $(P<0.05)$ in LP-CA than LP-FE, but not in C-CA and C-FE, and was lower $(P<0.05)$ in EM compared to $\mathrm{CA}$ and $\mathrm{FE}$ regardless of dietary treatment. Lower $(P<0.05)$ semitendinosus muscle weights were observed in C-CA compared to C-FE pigs, whereas no sex effect was observed in the LPgroups.

Predicted growth rates of the organs, gastrointestinal tract, semitendinosus muscle, and carcass cuts

The allometric regression coefficients determined for the weights of kidneys, liver, intestinal tract, semitendinosus muscle, carcass length and the different carcass components are presented in Table 2 . The $R^{2}$ of the allometric regression ranged from 0.895 (omental fat of LP-EM) to 0.997 (defatted carcass lean cuts of LP-EM and LP-FE). 
Ruiz-Ascacibar, Stoll and Bee

Table 1 Average and standard deviation [in brackets] of the transformed data of the weight of organs, gastrointestinal tract, carcass cuts and semitendinosus muscle from female (FE), castrated (CA) and entire male (EM) pigs fed either a control (C) or low-CP (LP) grower (20 to $60 \mathrm{~kg})$, finisher I (60 to $100 \mathrm{~kg})$ and finisher II $(100$ to $140 \mathrm{~kg})$ diet $^{1,2}$

\begin{tabular}{|c|c|c|c|c|c|c|c|}
\hline \multirow[b]{2}{*}{ Items } & \multicolumn{3}{|c|}{ C } & \multicolumn{3}{|c|}{$\mathrm{LP}$} & \multirow{2}{*}{$\begin{array}{l}\text { Orthogonal } \\
\text { contrasts }^{3}\end{array}$} \\
\hline & EM & CA & $\mathrm{FE}$ & EM & CA & $\mathrm{FE}$ & \\
\hline Kidneys (g) & $328[22.4]$ & 282 [12.4] & $291[14.5]$ & $305[22.5]$ & 282 [14.2] & 257 [12.3] & $b, c, d, e$ \\
\hline Liver (g) & $1557[90.4]$ & $1457[60.9]$ & $1482[80.0]$ & $1626[98.6]$ & $1393[61.9]$ & $1380[74.7]$ & $d$ \\
\hline $\begin{array}{l}\text { Gastrointestinal tract } \\
(\mathrm{g})\end{array}$ & $3723[191.8]$ & $3418[143.1]$ & $3582[181.5]$ & $3769[223.7]$ & $3392[147.9]$ & 3508 [193.2] & $c, d$ \\
\hline Carcass length (cm) & $92.0[2.41]$ & 90.6 [2.43] & $91.7[2.36]$ & $94.2[2.5]$ & $91.3[2.35]$ & $92.8[2.35]$ & $a, b, d, e$ \\
\hline Lean cuts ${ }^{4}(g)$ & 20006 [1609.4] & 19875 [1566.2] & $20145[1567.1]$ & 19707 [1686.1] & 19359 [1560.7] & 19554 [1620.2] & b, e \\
\hline Lean cuts ${ }^{5}(\%)$ & $61.8[0.4]$ & $60.0[0.55]$ & $61.0[0.57]$ & $59.9[0.45]$ & $58.5[0.53]$ & $60.4[0.48]$ & $a, b, c, e$ \\
\hline Loin (g) & $9258[788.1]$ & $9282[772.1]$ & $9457[777.1]$ & 9228 [829.9] & 9150 [782.7] & 9175 [797.9] & $\mathrm{e}$ \\
\hline $\operatorname{Ham}(\mathrm{g})$ & $6256[478.3]$ & $6179[474.7]$ & $6350[465.6]$ & $6113[498.1]$ & 5998 [477.7] & $6160[498.8]$ & $a, b, e$ \\
\hline Shoulder (g) & $4492[346.6]$ & $4381[324.8]$ & $4338[329.0]$ & $4367[362.3]$ & $4222[309.6]$ & $4217[329.2]$ & $\mathrm{e}$ \\
\hline Belly $(g)$ & $5972[523.6]$ & $6233[545.1]$ & $6278[555.3]$ & $6219[581.7]$ & $6327[556.9]$ & $6110[548.6]$ & $c, d$ \\
\hline Omental fat (g) & 763 [99.2] & $1153[181.4]$ & $1045[157.4]$ & 1065 [155.3] & 1325 [194.2] & 1142 [149.5] & $c, d, e$ \\
\hline Subcutaneous fat ${ }^{6}(\mathrm{~g})$ & $4552[464.6]$ & $5300[570.7]$ & $5124[562.7]$ & $5099[615.8]$ & 5611 [589.9] & 4815 [521.1] & $a, b, c, d, e$ \\
\hline Backfat $(\mathrm{g})$ & $2667[288.2]$ & $3184[365.6]$ & 2939 [346.3] & 3032 [399.3] & $3348[366.6]$ & 2848 [331.8] & $a, b, c, e$ \\
\hline Ham fat (g) & $834[81.9]$ & $1016[106.7]$ & 1015 [111.2] & 915 [104.6] & $1076[116.2]$ & 994 [108] & $b, c, d, e$ \\
\hline Shoulder fat (g) & 1051 [99.7] & $1100[104.3]$ & 1029 [104.1] & $1152[121]$ & $1186[110.1]$ & $974[88.5]$ & $a, b, e$ \\
\hline $\begin{array}{l}\text { Semitendinosus } \\
\text { muscle (g) }\end{array}$ & $388[36.1]$ & $382[34.9]$ & $412[31.8]$ & $366[31.5]$ & $368[32.2]$ & $382[32.5]$ & a \\
\hline
\end{tabular}

The values are estimated across the BW categories of $40,60,80,100,120$ and $140 \mathrm{~kg}$.

${ }^{1}$ The way to obtain the transformed data is detailed in the Supplementary Material S1.

${ }^{2} \mathrm{C}=$ formulated to meet nutrient requirements for grower-finisher pigs in the grower, finisher I and finisher II periods according to the Swiss feeding recommendations

for pigs; $L P=$ formulated to contain, expressed as percentage of $C$ diets, $80 \%$ of dietary $C P$, lysine, methionine + cystine, threonine and tryptophan.

${ }^{3}$ Significant $(P<0.05)$ orthogonal contrasts evaluated were $a=C-F E v . C-C A ; b=$ LP-FE $v$. LP-CA; $C=C-E M v$. C-FE/CA; $d=$ LP-EM v. LP-FE/CA; $=$ =C-FE/CA/EM v. LP-FE/

CA/EM $P<0.05$

${ }^{4}$ Sum of cold defatted loin, ham and shoulder weights obtained from the left carcass side.

${ }^{5}$ Sum of cold defatted loin, ham and shoulder weights expressed as weight percentage of the left carcass side.

${ }^{6}$ Sum of subcutaneous cold fat and skin without hairs from the shoulder, back and ham from the left carcass.

\section{Growth of organs and gastrointestinal tract}

The allometric coefficients $b$ for the kidneys, liver and GIT were, with $\leqslant 0.805$ markedly below 1 , implying a decreasing growth rate with increasing EBW (Table 2). Entire males fed the LP-diets had lighter $(P<0.05)$ kidneys, with slower $(P<0.05)$ growth rates than their $C$-counterparts. Kidneys of LP-CA and -FE were lighter $(P<0.05)$ and grew at a slower $(P<0.05)$ rate than those of $\mathrm{C}-\mathrm{CA}$ and $\mathrm{C}-\mathrm{FE}$ throughout the growth period (Figure 1a). Entire males of the LP-group had the greatest, while LP-CA and -FE pigs had the lowest, allometric coefficients $b$ and liver growth rates (Figure $1 \mathrm{~b}$ ) with intermediate values for C-EM, -CA and -FE. Regardless of dietary treatment, EM had heavier GIT above $36 \mathrm{~kg}$ EBW and a greater allometric coefficient than CA and FE-pigs. This led to the faster $(P<0.05)$ growth rates of GIT in EM when compared to CA and FE.

\section{Growth of primal cuts}

The lower a-value of the allometric regression together with the greater $b$-coefficient (Table 2) resulted in greater $(P<0.05)$ amounts of total lean cuts and greater $(P<0.05)$ deposition rate (above $38 \mathrm{~kg}$ EBW; Figure 1c) in LP-FE compared to C-pigs. Above 55 and $21 \mathrm{~kg} \mathrm{EBW}$, the amount and deposition rate of total lean cuts was greater $(P<0.05)$ in LP-CA than LP-EM. In addition, the greater allometric coefficient $b$ determined for LP-EM and LP-CA compared to $C$-pigs explains their slightly faster $(P<0.05)$ increase in total lean cut growth rate with increasing EBW. Nevertheless, as the a-values were lower in LP-EM and LP-CA than C-EM and $C-C A$, the total amount of deposited total lean cuts was lower $(P<0.05)$ throughout the growth period. The loin was the sole lean primal cut where $b$-coefficients were $>1$ (Table 2). Loin and shoulder weights and growth rates were, regardless of sex, greater $(P<0.05)$ in $C$ - than LP-pigs. However, with increasing EBW, differences in growth rates decreased between the experimental groups. Growth rates of ham and shoulder decreased with increasing EBW in all groups ( $b$-coefficient $<1)$. Above $39 \mathrm{~kg} \mathrm{EBW}$, increase in ham weight was greater $(P<0.05)$ in $\mathrm{LP}-\mathrm{FE}$ than $\mathrm{C}-\mathrm{FE}$, whereas greater $(P<0.05)$ growth rates were observed in $C-C A$ and C-EM when compared to their LP-counterparts. Belly weight and growth rate (Figure 1d) were greater $(P<0.05)$ in CAand FE- than EM-pigs, regardless of the dietary treatment.

\section{Growth of fat tissues}

Compared to all organs and carcass components monitored in the present study, omental fat displayed the greatest allometric $b$-coefficients, ranging from 1.576 (C-EM) to 2.174 
Growth dynamics of body components in pigs

Table 2 Allometric growth coefficients for weight of organs, gastrointestinal tract, carcass cuts and semitendinosus muscle from female (FE), castrated (CA) and entire male (EM) pigs fed either a control (C)or low-CP (LP) grower (20 to 60 kg), finisher I (60 to $100 \mathrm{~kg}$ ) and finisher II (100 to $140 \mathrm{~kg}) \mathrm{diet}^{7}$

\begin{tabular}{|c|c|c|c|c|c|c|}
\hline Items & Diet & Sex & a & $\mathrm{b}$ & $\mathrm{RSE}^{2}$ & $R^{23}$ \\
\hline \multirow[t]{5}{*}{ Kidneys (g) } & C & EM & 9.1 & 0.805 & 30.8 & 0.965 \\
\hline & C & $\mathrm{CA}, \mathrm{FE}$ & 18.7 & 0.611 & 25.5 & 0.961 \\
\hline & LP & EM & 11.4 & 0.735 & 37.7 & 0.936 \\
\hline & LP & CA & 19.6 & 0.582 & 27.3 & 0.949 \\
\hline & LP & $\mathrm{FE}$ & 19.4 & 0.582 & 23.4 & 0.960 \\
\hline \multirow[t]{3}{*}{ Liver (g) } & c & $\mathrm{EM}, \mathrm{CA}, \mathrm{FE}$ & 82.1 & 0.650 & 161.5 & 0.944 \\
\hline & LP & EM & 69.3 & 0.703 & 127.8 & 0.974 \\
\hline & LP & $\mathrm{CA}, \mathrm{FE}$ & 86.3 & 0.623 & 143.3 & 0.951 \\
\hline \multirow[t]{2}{*}{ Gastrointestinal tract $(\mathrm{g})$} & $C, L P$ & $\mathrm{EM}$ & 200.9 & 0.656 & 301.8 & 0.967 \\
\hline & $C, L P$ & $\mathrm{CA}, \mathrm{FE}$ & 261.6 & 0.582 & 308.5 & 0.957 \\
\hline \multirow[t]{6}{*}{ Carcass length (g) } & C & $\mathrm{EM}$ & 22.2 & 0.323 & 3.3 & 0.992 \\
\hline & C & CA & 21.9 & 0.322 & 2.2 & 0.995 \\
\hline & C & $\mathrm{FE}$ & 22.9 & 0.315 & 2.4 & 0.994 \\
\hline & LP & EM & 22.2 & 0.326 & 3.3 & 0.992 \\
\hline & LP & CA & 22.2 & 0.321 & 2.5 & 0.993 \\
\hline & LP & $\mathrm{FE}$ & 22.9 & 0.318 & 2.5 & 0.994 \\
\hline \multirow[t]{4}{*}{ Lean cuts $(\mathrm{g})^{4}$} & c & $\mathrm{EM}, \mathrm{CA}, \mathrm{FE}$ & 221.7 & 1.007 & 688.8 & 0.996 \\
\hline & LP & EM & 211.8 & 1.009 & 595.1 & 0.997 \\
\hline & LP & CA & 201.4 & 1.022 & 636.9 & 0.996 \\
\hline & LP & $\mathrm{FE}$ & 216.4 & 1.013 & 595.3 & 0.997 \\
\hline \multirow{2}{*}{ Loin $(g)$} & C & $\mathrm{EM}, \mathrm{CA}, \mathrm{FE}$ & 76.5 & 1.074 & 307.2 & 0.996 \\
\hline & LP & $\mathrm{EM}, \mathrm{CA}, \mathrm{FE}$ & 72.3 & 1.083 & 341.6 & 0.995 \\
\hline \multirow[t]{6}{*}{$\operatorname{Ham}(\mathrm{g})$} & c & EM & 78.7 & 0.979 & 209.4 & 0.996 \\
\hline & C & CA & 85.3 & 0.959 & 300.5 & 0.992 \\
\hline & C & $\mathrm{FE}$ & 101.5 & 0.927 & 301.3 & 0.992 \\
\hline & LP & EM & 83.4 & 0.957 & 206.9 & 0.996 \\
\hline & LP & CA & 76.0 & 0.978 & 251.4 & 0.994 \\
\hline & LP & $\mathrm{FE}$ & 82.2 & 0.971 & 294.4 & 0.992 \\
\hline \multirow{2}{*}{ Shoulder (g) } & C & $\mathrm{EM}, \mathrm{CA}, \mathrm{FE}$ & 63.6 & 0.949 & 218.5 & 0.991 \\
\hline & LP & $\mathrm{EM}, \mathrm{CA}$, FE & 64.7 & 0.939 & 1584.6 & 0.993 \\
\hline \multirow{2}{*}{ Belly (g) } & $C, L P$ & EM & 40.8 & 1.116 & 284.7 & 0.993 \\
\hline & $C, L P$ & $\mathrm{CA}, \mathrm{FE}$ & 40.4 & 1.127 & 256.9 & 0.994 \\
\hline \multirow[t]{4}{*}{ Omental fat (g) } & C & EM & 0.641 & 1.573 & 156.7 & 0.908 \\
\hline & C & $\mathrm{CA}, \mathrm{FE}$ & 0.056 & 2.174 & 255.5 & 0.903 \\
\hline & LP & $\mathrm{EM}$ & 0.528 & 1.678 & 238.5 & 0.895 \\
\hline & LP & $\mathrm{CA}, \mathrm{FE}$ & 0.372 & 1.796 & 261.7 & 0.915 \\
\hline \multirow[t]{6}{*}{ Subcutaneous fat $(g)^{5}$} & C & EM & 14.8 & 1.277 & 450.7 & 0.973 \\
\hline & C & CA & 9.1 & 1.417 & 442.8 & 0.982 \\
\hline & C & $\mathrm{FE}$ & 7.0 & 1.460 & 483.3 & 0.976 \\
\hline & LP & EM & 6.7 & 1.467 & 519.3 & 0.974 \\
\hline & LP & CA & 11.9 & 1.371 & 467.4 & 0.982 \\
\hline & LP & $\mathrm{FE}$ & 11.0 & 1.360 & 461.7 & 0.977 \\
\hline \multirow[t]{6}{*}{ Backfat (g) } & C & EM & 6.3 & 1.347 & 327.7 & 0.960 \\
\hline & C & CA & 3.1 & 1.540 & 322.9 & 0.975 \\
\hline & C & $\mathrm{FE}$ & 2.8 & 1.548 & 342.2 & 0.967 \\
\hline & LP & EM & 1.8 & 1.637 & 371.8 & 0.965 \\
\hline & LP & CA & 5.2 & 1.438 & 299.3 & 0.981 \\
\hline & LP & $\mathrm{FE}$ & 4.3 & 1.451 & 379.9 & 0.959 \\
\hline \multirow[t]{5}{*}{ Ham fat (g) } & C & EM & 4.3 & 1.179 & 105.8 & 0.953 \\
\hline & C & $\mathrm{CA}, \mathrm{FE}$ & 2.2 & 1.370 & 109.0 & 0.969 \\
\hline & LP & EM & 1.7 & 1.397 & 130.0 & 0.947 \\
\hline & LP & CA & 2.1 & 1.389 & 134.4 & 0.961 \\
\hline & LP & $\mathrm{FE}$ & 2.5 & 1.343 & 96.6 & 0.976 \\
\hline \multirow{3}{*}{ Shoulder fat (g) } & C & EM & 5.4 & 1.177 & 83.1 & 0.981 \\
\hline & C & CA & 5.8 & 1.173 & 100.5 & 0.975 \\
\hline & C & $\mathrm{FE}$ & 3.4 & 1.276 & 96.3 & 0.975 \\
\hline
\end{tabular}


Table 2 (Continued)

\begin{tabular}{lcccrrr}
\hline Items & Diet & Sex & a & b & RSE $^{2}$ & $R^{23}$ \\
\hline & LP & EM & 6.7 & 1.144 & 127.8 & 0.962 \\
& LP & CA & 6.1 & 1.176 & 80.8 & 0.987 \\
Semitendinosus muscle (g) & LP & FE & 6.3 & 1.130 & 85.8 & 0.977 \\
& C & EM & 3.1 & 1.071 & 34.9 & 0.969 \\
& C & CA & 2.8 & 1.098 & 46.0 & 0.954 \\
& C & FE & 4.9 & 0.984 & 42.9 & 0.959 \\
& LP & EM, CA, FE & 3.1 & 1.071 & 34.9 & 0.969 \\
\hline
\end{tabular}

${ }^{1}$ The allometric regression used was as follows: $Y=a \times \mathrm{EBW}^{b}$, where $Y$ is the predicted component weight $(\mathrm{g}$ or $\mathrm{cm})$; EBW the empty BW $(\mathrm{kg}) ; b$ the allometric coefficient; $a$ the constant; $R^{2}$ is based on the original and untransformed data.

${ }^{2}$ Residual standard error (RSE) $=[(\mathrm{SSE}) /(n-2)] 1 / 2$; where SSE is the error sum of squares; $n-2$ the degrees of freedom.

${ }^{3}$ Coefficient of determination.

${ }^{4}$ Sum of defatted loin, ham and shoulder weights obtained from the left carcass side.

${ }^{5}$ Sum of subcutaneous fat from the shoulder, back and ham from the left carcass side.

(a)

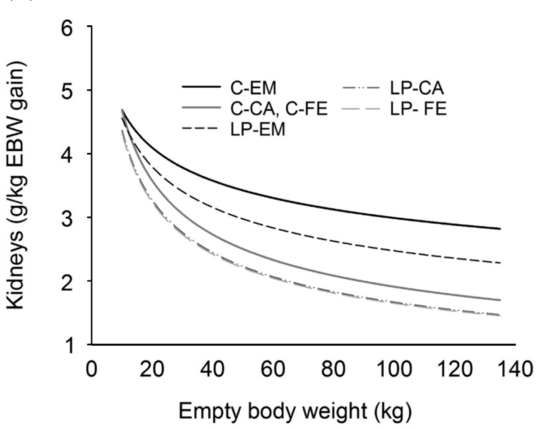

(d)

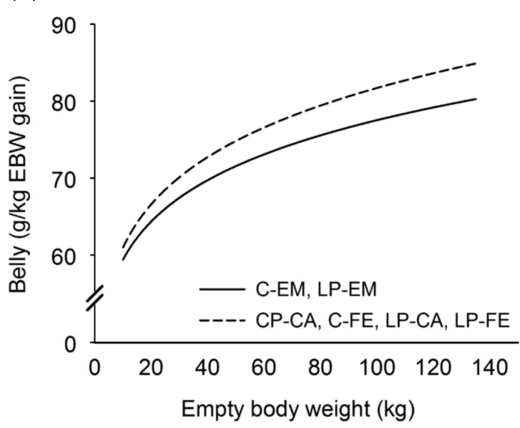

(b)

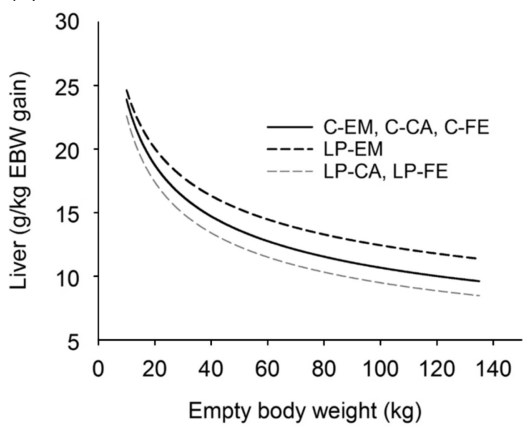

(e)

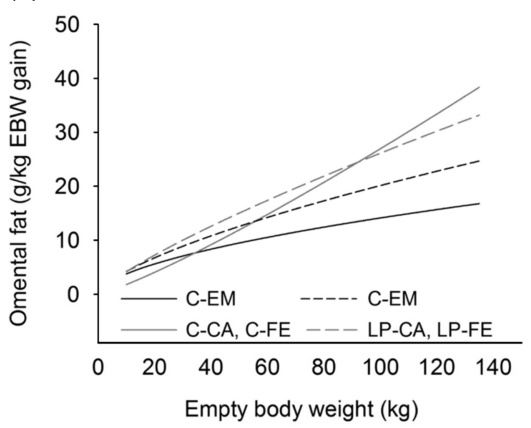

(c)

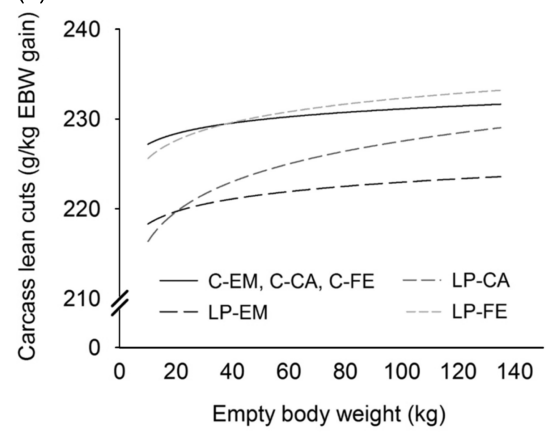

(f)

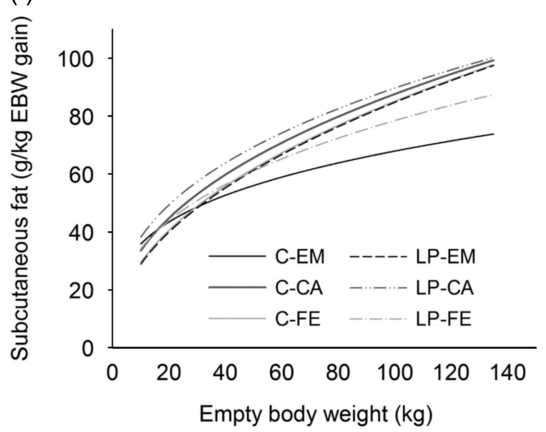

Figure 1 Dynamics of changes in relative growth rates ( $/ \mathrm{kg}$ empty BW gain) of the kidneys (a), liver (b), total weight of carcass lean cuts (c), belly (d), omental fat (e) and total subcutaneous fat (f) with increasing empty BW. To differentiate between treatment groups, the following orthogonal contrasts were used: FE v. CA fed either diet C (C-FE v. C-CA) or LP (LP-FE v. LP-CA); EM v. FE/CA fed either diet C (C-EM v. C-FE/CA) or LP (LP-EM v. LP-FE/CA); diet $\mathrm{C} v$. diet LP regardless of sex (C-FE/CA/EM v. LP-FE/CA/EM). Data of pigs from birth to $140 \mathrm{~kg}$ BW were used for calculating the allometric equations. $E M=$ entire males; $C A=$ castrated pigs; $F E=$ female pigs. Control diet $(C)$ grower $(20$ to $60 \mathrm{~kg})$, finisher I (60 to $100 \mathrm{~kg})$ and finisher II $(100$ to $140 \mathrm{~kg})$ diets were formulated to meet nutrient requirement according to the standard Swiss feeding recommendations for grower finisher pigs in the respective growth periods; low-CP diets (LP) were formulated to contain, expressed as a percentage of the control grower, finisher I and finisher II diets, $80 \%$ of dietary $\mathrm{CP}$, lysine, methionine + cystine, threonine and tryptophan.

(C-CA and FE) and therefore the greatest increase in growth rate (Table 2, Figure 1e). In C-CA and -FE pigs, a sharp increase in omental fat weight occurred in the finishers I and II periods (b-coefficient $=2.174$ ). This was caused by the steepest increase in growth rate when compared to all other groups (Figure 1e). Due to the lower $b$-coefficient in LP-CA and LP-FE pigs compared to their C-counterparts, differences in omental fat weight between both groups decreased with increasing EBW. Nevertheless, LP-CA and LP-FE deposited the greatest $(P<0.05)$ amount of omental fat of all experimental groups.

Backfat displayed the greatest allometric $b$-coefficients, followed by ham fat and shoulder fat. The greatest $(P<0.05)$ amount of back, ham, shoulder and total subcutaneous fats was determined in LP-CA pigs. Except for backfat above $87 \mathrm{~kg}$ EBW, this was true also for growth rates of these tissues. When compared with C-EM, LP-EM pigs had greater $(P<0.05)$ back, ham and subcutaneous fat contents above 
74,74 and $65 \mathrm{~kg}$ EBW, respectively. However, growth rates of back, ham and total subcutaneous fats were already greater above 38,35 and $32 \mathrm{~kg}$ EBW, respectively, in LP-EM than C-EM. In C-FE, the amount of back, ham, shoulder and total subcutaneous fat surpassed that of LP-FE above 95, 108, 74 and $91 \mathrm{~kg}$ EBW, respectively. However, EM growth rates of back, ham, shoulder and total subcutaneous fat were already greater above 50, 45, 32 and $44 \mathrm{~kg}$ EBW in C-FE than LP-FE.

\section{Growth of muscle}

Although C-FE had the heaviest semitendinosus muscle, growth rate decreased with increasing EBW. This is in contrast with muscle growth of the other experimental groups which displayed an allometric $b$-coefficient above 1 (Table 2). Thus, above $70 \mathrm{~kg}$ EBW semitendinosus muscle growth rate of C-EM, LP-EM, LP-CA and LP-FE surpassed that of C-FE.

\section{Discussion}

Some studies have reported growth dynamics of primal carcass cuts (Gu et al., 1992; Siewerdt et al., 1994; Landgraf et al., 2007; Nieto et al., 2013). However, comparisons between the growth dynamics of these traits across studies must be done carefully, because cutting standards vary, not only from country to country (Landgraf et al., 2007), but also between butchers (Nissen et al., 2006).

\section{Growth dynamics of organs and gastrointestinal tract} Reported weights for kidneys and liver at $101 \mathrm{~kg} \mathrm{BW}$ are consistent with data obtained earlier from the same breed (Pardo et al., 2013). The similar feed intake in the C- and LPgroups combined with the difference in dietary $\mathrm{CP}$ content between C- and LP-diets resulted in an overall lower amount of ingested protein in LP-pigs (Ruiz-Ascacibar et al., 2017). Since the kidneys and liver are major sites of AA metabolism and nitrogen clearance (Chen et al., 1999), their weight was expected to be greater in C-pigs due to the greater amounts of nitrogenous compounds to process. In accordance, kidneys were smaller in LP-pigs and the weight gain declined faster than in their C-counterparts. Furthermore, RuizAscacibar et al. (2017) reported that LP-pigs displayed a numerically greater $\mathrm{N}$-efficiency $(41.5 \mathrm{v} .45 .9)$ than C-pigs in the finisher period, supporting the idea of lower requirements for nitrogen clearance in LP than C-pigs. Schoknecht and Pond (1993) observed that the reason for dramatic weight increases in liver and kidneys of rats, after receiving twice the recommended supply of $\mathrm{CP}$, was hypertrophy, without concomitant hyperplasia. In line with the study of Moughan et al. (1990), kidney weight differed among sexes, being heavier in EM than FE and CA. In contrast to Chen et al. (1999) and Critser et al. (1995), who used five dietary CP levels ranging from $13 \%$ to $25 \%$ and $13 \%$ to $18.4 \%$ in their studies, dietary $\mathrm{CP}$ and essential AA levels in the current study did not influence liver weight. It is possible that diets in the present experiment were not sufficiently different to affect hepatic metabolism resulting in liver hypertrophy. The greater GIT weight in EM compared to CA and FE could be partly related to a greater absorptive capacity of the intestine, which would fit with the greater feed efficiency found in EM (Ruiz-Ascacibar et al., 2017).

\section{Growth dynamics of carcass lean cuts}

Percentage lean cuts obtained for C-CA (60\%) and C-FE $(62 \%)$ pigs at $100 \mathrm{~kg} \mathrm{BW}$ in the present experiment were greater than in the study of Bee et al. (2004) carried out with Swiss Large White pigs fed grower-finisher diets containing $18 \%$ and $13.5 \%$ CP and slaughtered at $105 \mathrm{~kg} \mathrm{BW}$ (CA: $55.3 \%$; FE: $57.3 \%$ ). Part of this difference can be explained by differences in the diets and/or genetic improvements in the last decades. In this regard, it is noteworthy mentioning that the greater percentage of lean cuts in the present study was a result of the greater relative weight of defatted loin and shoulder but not ham, because the relative ham weight was similar to values reported by Bee et al. (2004). The restriction in dietary $\mathrm{CP}$ and essential AA supply applied in the present study did not have the same impact on the weight of the individual primal cuts. Furthermore, EM, CA and FE showed different responses to the dietary treatment. Loin growth rate increased with increasing EBW in both $C$ and LP diets. However, at lower EBW, the growth rate was slower in the LP- than C-group, but this difference decreased with increasing EBW. The impact of the LP diet on the loin weight, which amounted to $175 \mathrm{~g}$ or $1 \%$ lower loin weight at $110 \mathrm{~kg}$ EBW, is of importance because loin is among the most popular carcass cuts in Europe (Verbeke et al., 2010). Thus, the economical relevance of the loin for the total carcass value is the greatest among all the three primal cuts (Marcoux et al., 2007). The difference between diets for the shoulder weight was $3 \%$ at $110 \mathrm{~kg}$ EBW. In contrast to shoulder and loin, dietary $\mathrm{CP}$ restriction had a different impact on the ham weight of the different sexes. The fastest reduction in defatted ham growth was observed in C-FE, which led to similar ham weights in C- and LP-groups at $110 \mathrm{~kg}$ EBW. However, C-EM and C-CA pigs had $5 \%$ and $3 \%$ heavier hams than their LP-counterparts, because the ham growth rate was greater in C-EM and C-CA pigs during all the analyzed growth periods.

\section{Growth dynamics of fat cuts}

The increase of BW was mainly linked to an increase in fat tissues ( $b$-coefficient: $\geqslant 1.144)$ rather than muscle ( $b$-coefficient: $\leqslant 1.083$ ), as observed by Siewerdt et al. (1994). Excessive carcass fat deposition is undesired because it lowers production efficiency, reduces the value of the carcass and increases labor for fat trimming at the abattoir (Lambe et al., 2013). Different responses to the dietary CP restriction were observed among sexes in terms of subcutaneous fat deposition rates. While LP-EM and LP-CA pigs accumulated fat at greater rates than their counterparts of the C-groups, LP-FE displayed lower subcutaneous fat deposition rates than C-FE in the finisher period. As a result, when the presented equations where used to predict 
subcutaneous fat content for a LP-EM and LP-CA pig at $110 \mathrm{~kg}$ EBW, the amount was $11 \%$ and $5 \%$ greater compared to C-EM and C-CA (639 and $378 \mathrm{~g}$, respectively). By contrast, the amount of subcutaneous fat was $2 \%(137 \mathrm{~g})$ lower in LP-FE than C-FE. Tous et al. (2014) found that pig's backfat thickness increased with dietary CP restriction but not with dietary lysine restriction. Thus, the increase in the amount of subcutaneous fat in LP-EM and LP-CA could be linked to the reduction of $C P$ rather to the essential $A A$ restriction.

Within sex growth dynamics of backfat, ham and shoulder fat differed. Regardless of sex, the fastest deposition rates were observed in backfat. This is in line with findings of Landgraf et al. (2007) obtained with Pietrain $\times$ (Large White $\times$ Landarce $\times$ Leicoma) CA and FE fed diets optimized for maximum protein deposition. In EM, ham fat deposition rate was lower than shoulder ham fat deposition rate. By contrast, in FE and $\mathrm{CA}$, ham fat deposition rates surpassed shoulder fat deposition rates in the grower and finisher period, respectively. The present findings are partly in contrast to those of Landgraf et al. (2007) who reported that shoulder fat deposition in FE and CA was almost constant $(b=1.05)$ and was the lowest among the three fat cuts.

Growth dynamics of total primal cuts, especially ham and loin, and of subcutaneous fat in LP-CA and LP-FE compared to the C-CA and C-FE is in line with the conclusions drawn from the determination of the dynamic of EB protein and fat content change reported by Ruiz-Ascacibar et al. (2019): a reduction of the dietary $\mathrm{CP}$ and essential AA supply might be positive if applied to the finisher period. Present results also support that the LP-diet did not allow LP-EM to express their full potential for lean growth.

\section{Conclusion}

Considering present and previous results reported for the same pigs regarding chemical deposition and feed efficiency and considering the potential cost reduction of the feed when CP restriction is applied, a compromise in terms of carcass composition might be worthwhile. A less restrictive diet in terms of $\mathrm{CP}$ and $\mathrm{AA}$ for the grower phase might be a solution to eliminate any deleterious effect on carcass cut growth dynamics, especially in EM and FE. Besides, a reduction of $C P$ supply only during the finisher phase is expected to lead to a better yield of lean primal cuts, as shown by its growth rate dynamics.

\section{Acknowledgements}

The authors acknowledge Guy Maïkoff and his team for taking care of the animals and his assistance during the slaughters; $\mathrm{Dr}$ Sébastien Dubois and his team for the chemical analysis of the diets; and Dr Paolo Silacci and his team for the help during sample collection.

\section{Declaration of interest}

The authors declare no conflicts of interest.

\section{Ethics statement}

The experimental procedure was approved by the Office for Food Safety and Veterinary Affairs (2012-14-FR 22119 and 2013-24-FR 24064) and all procedures were conducted in accordance with the Ordinance on Animal Protection and the Ordinance on Animal Experimentation.

\section{Software and data repository resources}

None of the data were deposited in an official repository.

\section{Supplementary material}

To view supplementary material for this article, please visit https://doi.org/10.1017/S1751731119000132

\section{References}

Agroscope 2017. Fütterungsempfehlungen für Schweine. Agroscope, Posieux, Switzerland. Retrieved on 18 October 2017 from www.agroscope.admin.ch.

Bee G, Gebert S and Messikommer R 2002. Effect of dietary energy supply and fat source on the fatty acid pattern of adipose and lean tissues and lipogenesis in the pig. Journal of Animal Science 80, 1564-1574.

Bee G, Guex G and Herzog W 2004. Free-range rearing of pigs during the winter: adaptations in muscle fiber characteristics and effects on adipose tissue composition and meat quality traits1. Journal of Animal Science 82, 1206-1218.

Chen HY, Lewis AJ, Miller PS and Yen JT 1999. The effect of excess protein on growth performance and protein metabolism of finishing barrows and gilts. Journal of Animal Science 77, 3238-3247.

Critser DJ, Miller PS and Lewis AJ 1995. The effects of dietary protein concentration on compensatory growth in barrows and gilts. Journal of Animal Science 73, 3376-3383.

Font-i-Furnols M, Čandek-Potokar M, Daumas G, Gispert M, Judas M and Seynaeve M 2016. Comparison of national ZP equations for lean meat percentage assessment in SEUROP pig classification. Meat Science 113, $1-8$.

Gispert M, Font-i-Furnols M, Gil M, Velarde A, Diestre A, Carrión D, Sosnicki AA and Plastow GS 2007. Relationships between carcass quality parameters and genetic types. Meat Science 77, 397-404.

Gu Y, Schinckel AP and Martin TG 1992. Growth, development, and carcass composition in five genotypes of swine. Journal of Animal Science 70, 1719-1729.

Kerr BJ and Easter RA 1995. Effect of feeding reduced protein, amino acidsupplemented diets on nitrogen and energy balance in grower pigs. Journal of Animal Science 73, 3000-3008.

Knecht D and Duziński $K$ 2016. The effect of sex, carcass mass, back fat thickness and lean meat content on pork ham and loin characteristics. Archives Animal Breeding 59, 51-57.

Knecht D, Duziński K and Lisiak D 2016. Accuracy of estimating the technological and economic value of pig carcass primal cuts with an opticalneedle device. Canadian Journal of Animal Science 96, 37-44.

Lambe NR, Wood JD, Mclean KA, Walling GA, Whitney H, Jagger S, Fullarton P, Bayntun J and Bunger $L$ 2013. Effects of low protein diets on pigs with a lean genotype 2. Compositional traits measured with computed tomography (CT). Meat Science 95, 129-136.

Landgraf S, Susenbeth A, Knap PW, Looft H, Plastow GS, Kalm E and Roehe R 2007. Developments of carcass cuts, organs, body tissues and chemical body composition during growth of pigs. Animal Science 82, 889-899. 
Marcoux M, Pomar C, Faucitano L and Brodeur C 2007. The relationship between different pork carcass lean yield definitions and the market carcass value. Meat Science 75, 94-102.

Mas G, Llavall M, Coll D, Roca R, Diaz I, Gispert M, Oliver MA and Realini CE 2010. Carcass and meat quality characteristics and fatty acid composition of tissues from Pietrain-crossed barrows and gilts fed an elevated monounsaturated fat diet. Meat Science 85, 707-714.

Moughan PJ, Smith WC and Stevens EVJ 1990. Allometric growth of chemical body components and several organs in the pig (20-90 kg liveweight). New Zealand Journal of Agricultural Research 33, 77-84.

Nieto R, Lara L, Barea R, Garcia-Valverde R, Conde-Aguilera JA and Aguilera JF 2013. Growth of body components and carcass composition of Iberian pigs of 10 to $150 \mathrm{~kg}$ body weight as affected by the level of feeding and dietary protein concentration. Journal of Animal Science 91, 4197-4207.

Nissen PM, Busk H, Oksama M, Seynaeve M, Gispert M, Walstra P, Hansson I and Olsen E 2006. The estimated accuracy of the EU reference dissection method for pig carcass classification. Meat Science 73, 22-28.

Pardo CE, Kreuzer $M$ and Bee $G$ 2013. Effect of average litter weight in pigs on growth performance, carcass characteristics and meat quality of the offspring as depending on birth weight. Animal 7, 1884-1892.

Ruiz-Ascacibar I, Stoll P, Kreuzer M and Bee G 2019. Dietary crude protein and amino acid restriction has a different impact on the dynamics of protein, amino acid and fat deposition in entire male, castrated and female pigs. Animal 13, $74-82$.
Ruiz-Ascacibar I, Stoll P, Kreuzer M, Boillat V, Spring P and Bee G 2017. Impact of amino acid and CP restriction from 20 to $140 \mathrm{~kg} \mathrm{BW}$ on performance and dynamics in empty body protein and lipid deposition of entire male, castrated and female pigs. Animal 11, 394-404.

Ruusunen M, Partanen K, Pösö R and Puolanne E 2007. The effect of dietary protein supply on carcass composition, size of organs, muscle properties and meat quality of pigs. Livestock Science 107, 170-181.

Schoknecht PA and Pond WG 1993. Short-term ingestion of a high protein diet increases liver and kidney mass and protein accretion but not cellularity in young pigs. Proceedings of the Society for Experimental Biology and Medicine $203,251-254$

Siewerdt F, Farias JVdS, Osório JCdS and Jacondino IFR 1994. Allometric growth of primal cuts and tissues in the pig. Ciência Rural 24, 623-627.

Tous N, Lizardo R, Vilà B, Gispert M, Font-i-Furnols M and Esteve-Garcia E 2014. Effect of reducing dietary protein and lysine on growth performance, carcass characteristics, intramuscular fat, and fatty acid profile of finishing barrows. Journal of Animal Science 92, 129-140.

Verbeke W, Pérez-Cueto FJA, Barcellos MDd, Krystallis A and Grunert KG 2010. European citizen and consumer attitudes and preferences regarding beef and pork. Meat Science 84, 284-292.

Wiseman TG, Mahan DC, Peters JC, Fastinger ND, Ching S and Kim YY 2007. Tissue weights and body composition of two genetic lines of barrows and gilts from twenty to one hundred twenty-five kilograms of body weight. Journal of Animal Science 85, 1825-1835. 